\title{
Características físicas y fisiológicas de los deportistas de orientación de alto rendimiento
}

\section{Physical and physiological characteristics of the high performance orienteers}

JOAQUÍN FIDALGO DÍAZ

IES Mata Jove. Consejería de Educación-Principado de Asturias. España xuacufidalgo@hotmail.com

Recibido: 20-03-2020. Aceptado: 04-06-2020.

Cómo citar /Citation: Fidalgo, J. (2020). Características físicas y fisiológicas de los deportistas de orientación de alto rendimiento, Ágora para la Educación Física y el Deporte, 22, 209-219.

DOI: https://doi.org/10.24197/aefd.0.2020.209-219

Resumen. En el deporte de orientación, ante la ausencia de estudios específicos acerca de los factores que determinan el rendimiento competitivo, se hace necesario recurrir a los datos de corredores de orientación de alto rendimiento. El presente artículo, aun reconociendo la importancia del factor psicológico en el rendimiento, pretende recabar los datos físicos y fisiológicos que definen a esta selecta población de deportistas.

Palabras clave. Deporte de orientación; consumo máximo de oxígeno; umbral anaeróbico; antropometría.

Abstract: In the sport of orienteering, due to the lack of specific studies about performance analysis, it is needed to draw on high level orienteers data. This paper, even recognizing the psychological importance in competition success, aims to collect physical and physiological data which define this select group of athletes.

Keywords: Orienteering; maximal oxygen consumption; anaerobic threshold; anthropometry.

\section{INTRODUCCIÓN}

La valoración de las capacidades de los deportistas de alto nivel es un dato muy relevante para conocer qué sistemas orgánicos están más implicados en deporte de orientación. 
El presente artículo se basa en una revisión bibliográfica para recopilar y ordenar los datos publicados hasta la fecha, y llegar a conclusiones que puedan aportar información novedosa para investigadores y entrenadores especializados en deporte de orientación. En este sentido, se ha recurrido a los datos de estudios sobre poblaciones de élite así como a las comparaciones entre éstos y otras poblaciones (sub-élite y amateur).

\section{MÉTODo}

Se ha llevado a cabo un triple filtro de búsqueda, de la siguiente manera:

Primer filtro. Se ha basado en teclear el operador booleano "orienteering" en la base de datos EBSCO para encontrar todo tipo de documentos, desde 1985 hasta 2019, que incluyeran esta palabra en sus textos. En total, 2152 documentos encontrados.

Las razones para establecer un primer filtro tan genérico son las siguientes:

- Escasez de publicaciones científicas que abordan el deporte de orientación $\mathrm{y}$, por tanto, necesidad de abrirse a otros documentos que puedan ser consultados. Por ejemplo, libros y artículos de revistas publicados por personas cualificadas (deportistas de alto nivel, técnicos, preparadores físicos, licenciados en Ciencias de la Actividad Física y el Deporte, etc.). Revistas como "Inside Orienteering", "Orienteering World", "Australian Orienteer", "Ozone" o "Compass Sport", aunque no siempre se basan en procedimientos científicos, nos aportan información puntual relevante debido a que tienen como campo de actuación el deporte de alto rendimiento.

- En el deporte de orientación, como se tratará más adelante, existe una gran relación entre los factores físicos, fisiológicos y psicológicos. Por esta razón, es necesario comprender el deporte de orientación desde diversos puntos de vista, no sólo desde el enfoque físico-fisiológico sobre el que versará el presente trabajo.

- Se ha escogido el periodo de 1985 a 2019 (última búsqueda actualizada a 15 de junio de 2019) por dos motivos:

a) Tras la formación en 1983 del primer grupo científico en torno al deporte de orientación (coordinado por la Federación Internacional de Orientación), Seiler (1986) lleva a cabo la primera revisión bibliográfica 
que recopila y ordena todas las publicaciones hasta 1985. Por lo tanto, el trabajo previo a esta fecha ya está realizado, dando como resultado 93 publicaciones entre 1967 y 1985.

b) Aparición, en 1985, de la revista "Scientific Journal of Orienteering" que, aunque no cuenta con factor de impacto, se basa en el método científico y aglutina la mayor parte de publicaciones sobre el deporte de orientación.

Segundo filtro. Tras la lectura de los títulos y/o abstracts al finalizar la primera búsqueda, se han rechazado todos aquellos artículos que son considerados irrelevantes. Esto se debe a que la calidad y/o fiabilidad es baja o bien porque no entran dentro de uno de los siguientes campos de estudio relacionados con el deporte de orientación:

- Análisis de competiciones.

- Antropometría.

- Biomecánica.

- Carga externa.

- Cartografía.

- Diferencias sexo/edad.

- Entrenamiento.

- Estilos de vida y personalidad.

- Fatiga y errores.

- Habilidad perceptiva y cognitiva.

- Impacto en flora y fauna.

- Lesiones.

- Monitorización de parámetros fisiológicos.

- Motivaciones de práctica.

- Nutrición.

- Orientación en principiantes.

- Procesos psicológicos.

- Revisiones bibliográficas.

Tras la aplicación de este segundo filtro se han listado y ordenado 227 publicaciones.

Tercer filtro. Para la elaboración de este trabajo se han establecido los siguientes criterios de inclusión:

- Artículos publicados en revistas que, aunque no tuvieran impacto científico, sí emplearan este método en sus investigaciones y fueran relevantes para definir los factores de rendimiento. Se han incluido 32 
artículos de revistas, de las cuales 16 son de impacto ( 5 en el primer cuartil, 6 en el segundo, 2 en el tercero y 3 en el cuarto).

- Artículos que, aunque no guarden una relación directa con el deporte de orientación, tras ser citados en varios artículos, se incorporan a la bibliografía debido a la relevancia que tienen para alcanzar los objetivos marcados.

- Escritos en inglés, castellano, francés o portugués. De los 37 documentos consultados, 35 están escritos en inglés y 2 en castellano.

- Escritos entre 1985 y el 31 de agosto de 2015. Excepción: un informe de 1980, citado en otro artículo consultado y necesario para completar el apartado relativo al consumo de oxígeno en deporte de orientación.

- Otros documentos (5) necesarios para completar el estudio: ponencias (1), tesis doctorales inéditas (1), informes (1), reglamentos de competición (1) y un archivo web que almacena resultados históricos de los WOC (1).

También es preciso señalar que, en ciertas ocasiones, se ha optado por hacer referencia exclusivamente a los valores obtenidos por los corredores de élite varones. Esto se debe, simplemente, al mayor número de estudios dirigidos a esta población.

\section{RESULTADOS}

\section{Consumo de Oxígeno Máximo y Umbral Anaeróbico}

Desde que Adams y Saltin (1980) monitorizaran un total de 39 corredores de orientación de alto rendimiento suizos, suecos $\mathrm{y}$ finlandeses hasta los trabajos de Tønessen (2015), se han sucedido hasta 20 estudios donde se ofrecían datos sobre el máximo rendimiento respiratorio de estos deportistas.

Aunque Smekal et al. (2003) habían recogido todos los datos hasta 2003, en el presente estudio se han encontrado ciertos datos de baja fiabilidad en dicha investigación, ya sean datos erróneos o muestras poco representativas al haber considerado a deportistas amateurs en sus investigaciones.

Por esta razón y a través de un exhaustivo estudio, se han recopilado todos los datos sobre el $\mathrm{VO}_{2 \text { máx }}$ y/o el UAN de los deportistas de élite masculino que aparecen en la literatura. 
Tabla I. Consumos de oxígeno $(\mathrm{ml} / \mathrm{kg} / \mathrm{min})$ y umbral anaeróbico (\% sobre el VO2máx) en orientadores élite masculino

\begin{tabular}{|l|l|l|l|l|l} 
Autor/es & Año & Nacionalidad & $\mathbf{N}$ & $\mathbf{V O}_{2 \text { máx }}$ & UAN \\
\hline Adams y Saltin & 1980 & $\begin{array}{l}\text { Suiza } \\
\text { Suecia }\end{array}$ & 5 & $68.5 \pm 2.5$ & \\
\hline Ranucci et al. & 1986 & Italia & 9 & $75.7 \pm 3.7$ & \\
\hline Johansson et al. & 1987 & Suecia & 14 & $69.6 \pm 3.6$ & \\
\hline Chalopin & 1994 & Francia & 14 & $71.7 \pm 5.7$ & $87.4 \pm 3.0$ \\
\hline Jensen et al. & 1994 & Dinamarca & 5 & $74.3 \pm 3.5$ & \\
\hline Moser et al. & 1995 & Noruega & 16 & $71.7 \pm 8.4$ & \\
\hline Held y Müller & 1997 & Suiza & 12 & $74.0 \pm 5.3$ & \\
\hline Gjerset et al. & 1997 & Noruega & 9 & $77.5 \pm 3.9$ & $84.9 \pm 5.1$ \\
\hline Rolf et al. & 1997 & Suecia & 5 & $78.4(75-81)$ & \\
\hline Jensen et al. & 1999 & Dinamarca & 11 & $73 \pm 2.0$ & \\
\hline Ladyga et al. & 2000 & Polonia & 4 & $76.6 \pm 4.8$ & $85.9 \pm 4.3$ \\
\hline Larsson et al. & 2002 & Suecia & 10 & $74.1 \pm 7.0$ & $88.0 \pm 9.9$ \\
\hline Smekal et al. & 2003 & Austria & 11 & $67.9 \pm 3.8$ & $87.7 \pm 5.1$ \\
\hline Ladyga et al. & 2004 & Polonia & 10 & $77.1 \pm 4.0$ & $84.6 \pm 5.4$ \\
\hline Tvezkov & 2009 & Bulgaria & 10 & $66.7 \pm 5.5$ & \\
\hline Rattray y Roberts & 2012 & Australia & 7 & $72.4 \pm 3.1$ & \\
\hline Sainz y Rabadán & 2013 & España & 5 & $71.4 \pm 8.0$ & $90.1 \pm 3.4$ \\
\hline Lauenstein et al. & 2013 & Suiza & 10 & $69.2 \pm 5.7$ & 92.7 \\
\hline Hébert-Losier et al. & 2015 & Suecia & 7 & $68.5 \pm 2.1$ & \\
\hline Tonessen et al. & 2015 & Noruega & 6 & $83(81-85)$ & \\
\hline & & & & & \\
\hline
\end{tabular}

Como podemos comprobar, los altos niveles de $\mathrm{VO}_{2 \text { máx }}$ y el UAN situado a un alto porcentaje del mismo están presenten en los orientadores de élite.

El mayor valor registrado es el que ofrecen Tønessen et al. (2015), cuyo criterio de inclusión en el estudio se basaba en orientadores noruegos de élite internacional que hubieran ganado durante esa temporada por lo menos una medalla en el WOC.

El menor valor lo ofrecen Ranucci, Grassi, Miserocchi et al. (1986) que centraron su estudio en orientadores de élite italianos, de bajo 
rendimiento internacional (en aquel WOC el primer orientador italiano ocupó el puesto 58 de la general).

Entre ambos estudios hay una diferencia de $26 \mathrm{ml} / \mathrm{kg} / \mathrm{min}$. Este dato, aunque debe ser analizado con cautela debido a la profesionalización del deporte en la actualidad (1986 vs 2015), muestra las diferencias existentes entre aquellos que ganan medallas y los que aspiran simplemente a meterse en las rondas finales.

Pablos (2005) también aportó información relevante, estudiando las diferencias de $\mathrm{VO}_{2 \text { máx }}$ entre orientadores de élite internacional y nacional masculino (tabla I). Llegó a la conclusión que el $\mathrm{VO}_{2 \text { máx }}$ es mayor en los deportistas de élite internacionales que en los nacionales, hecho que no ocurrió con los valores relativos al UAN donde, siendo mayores, no fueron estadísticamente significativos. Es interesante apuntar que los corredores de orientación españoles, aun participando en competiciones internacionales, no obtienen resultados significativos en las mismas.

Tabla II. Comparación del $\mathrm{VO}_{2 \text { máx }}$ y del UAN en orientadores de élite internacional y nacional masculino españoles. Modificado de Pablos (2005)

\begin{tabular}{l|l|c|l} 
Parámetro & Deportistas & Muestra & Media \\
VO $_{2 \text { máx }}$ & Élite internacional & 5 & $71,35 \pm 5,85$ \\
& Élite nacional & 11 & $61,06 \pm 3,80$ \\
\hline \% UAN & Élite internacional & 5 & $86,82 \pm 5,69$ \\
& Élite nacional & 11 & $84,53 \pm 5,37$
\end{tabular}

\section{Datos antropométricos}

En el presente estudio se han recogido y organizado los datos relativos al peso, la talla y el índice de masa corporal desde 1994 hasta la actualidad (tabla III). 
Tabla III. Talla (cm), peso ( $\mathrm{kg}$ ) e indice de masa corporal (IMC) en orientadores de alto rendimiento

\begin{tabular}{l|l|l|l|l|l|l} 
Publicación & Año & País & $\mathbf{N}$ & Talla & Masa & IMC \\
Chalopin & 1994 & Francia & 14 & $178,5 \pm 4,2$ & $66,4 \pm 5.6$ & $20,9 \pm 1,5$ \\
Moser & 1995 & Noruega & 16 & $181,7 \pm 5$ & $69,8 \pm 6.1$ & 21,1 \\
\hline Ladyga & 2000 & Polonia & 10 & & $65,6 \pm 6,6$ & \\
\hline Larsson & 2002 & Suecia & 10 & $187 \pm 8$ & $72,9 \pm 5,5$ & 20,8 \\
\hline Ladyga & 2004 & Polonia & 10 & & $63,1 \pm 3,5$ & \\
\hline Pablos & 2005 & España & 17 & $175,9 \pm 7,59$ & $69,4 \pm 7,93$ & 22,4 \\
\hline Zürcher & 2005 & Suiza & 9 & $179,6 \pm 7,3$ & $69,2 \pm 6,65$ & $21,4 \pm 1,0$ \\
\hline Tzvetkov & 2009 & Bulgaria & 10 & $180,0 \pm 6,7$ & $69,2 \pm 5,35$ & $21,1 \pm 2,0$ \\
\hline Rattray & 2012 & Australia & 7 & $182,0 \pm 0,1$ & $71,3 \pm 5.1$ & 21,6 \\
\hline Lauenstein & 2013 & Suiza & 10 & $180,7 \pm 6,7$ & $71,2 \pm 5.6$ & $21,8 \pm 0,6$ \\
\hline Hébert-Losier & 2014 & Suecia & 8 & $183,1 \pm 6,3$ & $71,9 \pm 6,0$ & 21,6 \\
\hline Tønnessen & 2015 & Noruega & 6 & $181(177-185)$ & $71(56-78)$ & 22,1 \\
\hline
\end{tabular}

Además de estos datos podemos incluir dos estudios interesantes. En primer lugar, Pablos (2005) comparó la composición corporal de los orientadores de élite internacional y nacional españoles, concluyendo lo siguiente:

Porcentaje de grasa corporal: élite internacional, $10.40 \%$ y élite nacional $11,73 \%$.

Masa muscular: 48,42\% para los de élite internacional y 47,64\% para los de élite nacional.

IMC. Valores muy similares.

Por su parte, Creagh y Reilly (1997) demostraron que las orientadoras de élite tenían unos valores de grasa corporal que eran un $19 \%$ más altos que los de las corredoras de asfalto.

\section{CONCLUSIONES}

La primera y más clara conclusión es que el corredor de orientación de élite presenta valores significativamente más altos de $\mathrm{VO}_{2 \text { máx }}$ respecto a otros corredores de menor nivel. Por lo tanto, un metabolismo aeróbico muy desarrollado se considera básico para rendir en este deporte. 
En segundo lugar, aunque con un poco menos de significatividad, observamos Umbrales Anaeróbicos a un porcentaje elevado respecto al $\mathrm{VO}_{2 \text { máx. }}$. Este dato resulta muy claro en aquellos corredores que alcanzan los máximos resultados en la modalidad sprint (12-15 minutos corriendo al máximo), no siendo tan evidente en los que se especializan en media y larga distancia, debido al carácter discontinuo de la competición, así como las pausas que se intercalan durante la misma.

En cuanto a los datos antropométricos, observamos que la altura media de los corredores de élite varones se sitúa por encima de $180 \mathrm{~cm}$, lo cual permite sugerir la importancia de una zancada relativamente amplia para la carrera a pie por diferentes superficies.

El peso se suele situar en torno a los 70-72 kilos que, relacionado con la altura media, nos da como resultado Índices de masa corporal relativamente bajos, en torno a 21 . Por tanto, un peso bajo respecto a la altura es un requisito básico para cualquier corredor que quiera obtener resultados en este deporte. Un kilo de más supone un lastre que habrá que acarrear durante pruebas que pueden alcanzar los 90 minutos de duración.

Aunque no existen muchos datos sobre la composición corporal, estos corredores suelen tener unos niveles de fuerza y una mayor hipertrofia muscular que el resto de atletas de resistencia. Esto se debe a las características particulares del deporte, tales como: superar desniveles positivos y negativos, saltos durante la carrera para salvar obstáculos, superficies que provocan mayor tiempo de contacto del pie y, en la modalidad sprint, aceleraciones y sprints durante gran parte de la prueba.

\section{BIBLIOGRAFIA}

Adams, D., \& Saltin, B. (1980). Physical work capacity in orienteering. International Orienteering Federation Report, 2, 9-11.

Chalopin, C. (1994). Physical and physiological characteristics of french orienteers. Scientific Journal of Orienteering, 10, 58-62.

Gjerset, A., Johansen, E., \& Moser, T. (1997). Aerobic and anaerobic demands in short distance orienteering. Scientific Journal of Orienteering, 13, 4-25.

Hébert-Losier, K., Jensen, K., Mourot, L., \& Holmberg, H. C. (2014). The influence of surface on the running velocities of elite and amateur athletes. 
Scandinavian Journal of Medicine and Science in Sports, 24(6), 448-455. DOI: $\underline{10.1111 / \mathrm{sms} .12224}$

Hébert-Losier, K., Platt, S., \& Hopkins, W. (2015). Sources of variability in performance times at the World Orienteering Championships. Medicine and Science in Sports and Exercise, 47(7), 1523-1529. DOI: 10.1249/MSS.0000000000000558

Held, T., \& Müller, I. (1997). Endurance capacity in orienteering - New field test vs. laboratory test. Scientific Journal of Orienteering, 13, 26-37.

Jensen, K., Franch, J., Kärkkäinen, O., \& Madsen, K. (1994). Field measurements of oxygen uptake in elite orienteers during cross-country running using telemetry. Scandinavian Journal of Medicine and Science in Sports, 4(4), 234-238. DOI: 10.1111/j.1600-0838.1994.tb00433.x

Jensen, K., Johansen, L., \& Kärkkäinen, O. (1999). Economy in track runners and orienteers during path and terrain running. Journal of Sports Sciences, 17(12), 945-950. DOI: $10.1080 / 026404199365335$

Johansson, C., Gerdle, B., Lorentzon, R., Rasmuson, S., Reiz, S., \& FugiMeyer, A. (1987). Fatigue and endurance of lower extremity muscles in relation to running velocity at OBLA in male orienteers. Acta Physiologica Scandinavica, 131(4), 203-209. DOI: 10.1111/j.1748-1716.1987.tb08227.x

Ladyga, M., Faff, J., Starczewska-Czapowska, J., \& Jarosiński, S. (2000). Physical fitness of the elite orienteers from the polish national team in the period from 1993 to 1998. Biology of Sport, 17(1), 25-35.

Ladyga, M., Faff, J., Starczewska-Czapowska, J., \& Jarosiński, S. (2004). Development of the aerobic fitness in elite young orienteers. Biology of sport, 21(2), 105-120.

Larsson, P., Burlin, L., Jakobsson, E., \& Henriksson-Larsén, K. (2002). Analysis of performance in orienteering with treadmill tests and physiological field tests using a differential global positioning system. Journal of Sports Sciences, 20, 529-535. DOI: $\underline{10.1080 / 026404102760000035}$

Lauenstein, S., Wehrlin, J. P., \& Bernard, M. (2013). Differences in horizontal vs. uphill running performance in male and female Swiss world-class 
orienteers. Journal of Strenght and Conditioning Research, 27(11), 29522958. DOI: $10.1519 / \mathrm{JSC} .0 \mathrm{~b} 013 \mathrm{e} 31828 \mathrm{bf} 2 \mathrm{dc}$

Moser, T., Gjerset A., Johansen E., \& Vadder, L. (1995). Aerobic and anaerobic demands in orienteering. Scientific Journal of Orienteering, 11, 3-30.

Pablos, A. (2005). Valoración de las capacidades físicas y cognitivas en corredores de orientación de la categoría hombres-élite. (Tesis doctoral inédita). Departamento de Educación Física y Deportiva. Universidad de Valencia. Disponible en: https://roderic.uv.es/handle/10550/23332

Ranucci, M., Grassi, G., \& Miserocchi, G. (1986). Anaerobic threshold in orienteers as an index of the aerobic-anaerobic relative contributions to the total power output - a comparison with other endurance sports. Scientific Journal of Orienteering, 2, 124-133.

Rattray, B. \& Roberts, A. (2012). Athlete assessments in orienteering: differences in physiological variables between field and laboratory settings. European Journal of Sport Science, 12(4), 293-300. DOI: $\underline{10.1080 / 17461391.2011 .566366}$

Rolf, C., Andersson, G., Westblad, P. \& Saltin, B. (1997). Aerobic and anaerobic work capacities and leg muscle characteristics in elite orienteers. Scandinavian Journal of Medicine and Science in Sports, 7(1), 20-24. DOI: 10.1111/j.1600-0838.1997.tb00112.x

Sainz, L. y Rabadán, M. (2013). Valoración de la condición aeróbica del corredor de orientación a pie de alto nivel español. Archivos de Medicina del Deporte, 30(6), 359-364. Obtenido el 15 de noviembre de 2018 en: http://archivosdemedicinadeldeporte.com/articulos/upload/or04_158.pdf

Smekal, G., Von Duvillard, S., Pokan, R., Lang, K., Baron, R., Tschan, H., Hofmann, P., \& Bachl, N. (2003). Respiratory gas exchange and lactate measures during competitive orienteering. Medicine and Science in Sports \& Exercise, 35(4), 682-89. DOI: 10.1249/01.MSS.0000058358.14293.DE

Tønnessen, E., Svendsen, I., Rønnestad, B., Hisdal, J., Haugen, T. A., \& Seiler, S. (2015). The annual training periodization of 8 world champions in orienteering. International Journal of Sports Physiology and Performance, 10, 29-38. DOI: $10.1123 /$ ijspp.2014-0005 
Tzvetkov, S. (2009). The reproducibility of the ventilatory anaerobic threshold determination methods among two maximal treadmill exercise protocols in elite orienteers. Facta Universitatis Series Physical Education and Sport, 7(1), 45-53. En: http://facta.junis.ni.ac.rs/pe/pe200901/pe200901-05.pdf

Zürcher, S., Clénin, G., \& Marti, B. (2005). Uphill running capacity in Swiss elite orienteers. Scientific Journal of Orienteering, 16, 41-58. 